\title{
Cross flow induced vibration in a single tube of square array using LES
}

\author{
Vilas Shinde, Elisabeth Longatte, Franck Baj, Yannick Hoarau, Marianna Braza
}

\begin{abstract}
Large eddy simulations (LES) of a single phase water flow through a square normal tube bundle at Reynolds numbers from 2000 to 6000 is performed to investigate the fluid-elastic instability. A single cylinder of the array is allowed to oscillate in one degree of freedom (1-DOF) in the flow normal direction, similar as in the corresponding experiments. The fluid-structure coupling is simulated using the Arbitrary Lagrangian-Eulerian (ALE) approach. The subgrid scale turbulence is modeled using the standard Smagorinsky's eddy-viscosity model. The LES results show a good agreement with the experimental results, in terms of the response frequency and damping ratio of the cylinder vibration. The dynamic case simulations are compared with static cases over the range of Reynolds numbers by means of the pressure profiles on the cylinder surface and the probe velocity spectra.
\end{abstract}

\section{Introduction}

Heat exchangers are vital component of the energy industries. The flow of fluids through the heat exchanger tube bundles can induce vibrations and may result in severe breakdown of the tubes. The cross flow induced vibrations in tube arrays are classified mainly in four categories, namely: turbulent buffeting, vibrations due to flow periodicity (or vortex induced vibrations), acoustic vibrations and fluid-elastic instability. The fluid-elastic instability is most devastating compared to the rest, yet it is less understood. A substantial amount of research work is conducted in order

Vilas Shinde, Elisabeth Longatte, Franck Baj

IMSIA, EDF-CNRS-CEA-ENSTA UMR 9219, 7, blvd Gaspard Monge 91120 Palaiseu, France

e-mail: vilas.j.shinde@gmail.com

Yannick Hoarau

ICUBE Laboratory, 2 Rue Boussingault, F - 67000 Strasbourg, France

Marianna Braza

IMFT, CNRS/INPT UMR 5502, Av. du prof. Camille Soula, 31400 Toulouse, France 
to enhance the understanding of the stability limit criteria, and thereby prevent its occurrence. Many theoretical models are developed since the phenomenon was first brought to notice by Roberts [4] and later by Connors [7]. The models proposed for the phenomenon include Blevins [5], Tanaka et al. [9], Chen et al. [19,20], Paidoussis and Price [13], Lever and Weaver [10], Granger et. al [8] etc.. Thus providing an insight into the fluid-elastic instability by means of different instability mechanisms such as 'fluid flow jet switching mechanism', 'stiffness controlled mechanism', 'damping controlled mechanism' and so on. In addition, the dynamic features of the instability such as the phase lag between fluid force and displacement, flow cell with boundary layer effect are considered while modeling the phenomenon. Although many approaches exist, all fall short individually to predict the phenomenon in all situations for wide range of parameters.

In addition to the experiments, Computational Fluid Dynamics (CFD) provides a possibility to simulate and better understand such phenomena. In the industrial context, Direct Numerical Simulation (DNS) is computationally very expensive. Although, the URANS approach is computationally less expensive, the transient features of the fluid flow and fluid-structure interaction are not well captured. The Large Eddy Simulation (LES) approach overcomes the shortcoming of URANS approach. Furthermore, contrary to the DNS approach, LES is computationally less expensive. The large scale transient flow is directly captured in LES, while as the small scale (subgrid scale) turbulence is modeled. Some of the early works on simulation of the flow through tube bundles using LES include the work of Hassan and Barsamian (1997, 1999). Further, the work of Rollet-Miet et al [15], Benhamadouche and Laurence [17] and C. Liang and G. Papadakis [6] confirmed the benefits of LES over URANS in the tube bundle configurations. Although Large Eddy Simulation (LES) is still expensive for high Reynolds number flows, it is well suited for the low Reynolds number flows. Recently, similar benefits of LES were reported for the vortex-induced vibrations at a moderate Reynolds number in Jus et. al. [11].

In present work, LES of a single phase cross flow through a square normal tube array is performed, in order to investigate the fluid-elastic instability. The Reynolds numbers are in a range from 2000 to 6000 approximately. The vibration in a single cylinder of the array is simulated by the Arbitrary Lagrangian Eulerian (ALE) approach. In many experiments, it has been observed that the fluid-elastic vibrations are pre-dominant in the flow normal direction, especially in water-flow experiments (Price et al. [18]). Also there are several studies performed on a single cylinder oscillating in a fixed cylinders arrangement (Price et al. [18], Khalifa et al. [1]). It leads to essentially the same critical velocities as for the fully flexible array of cylinders. Therefore the central cylinder is only monitored and allowed to oscillate in flow normal direction. Although, in some studies such as Kevlahan [14], the instability is found to be dominant in in-flow direction for wide range of mass-damping parameter and the critical velocities predicted using a single cylinder in fixed array are overestimated compared to the fully flexible array. The length of computational domain in spanwise direction is taken as $4 D$, which provides enough space for LES vortex dynamics. The article is arranged as follows: configuration of the flow, simulation results and analysis. 


\section{Configuration}

In experiments, the tube bundle $(5 \times 5)$ is located in a vertical flow channel. The cylinders are in square normal arrangement. The two side columns are half wall mounted. Only the cylinder at center is flexibly mounted, while the remaining cylinders are fixed. The channel depth (the length of cylinders) and width are $100 \times 10^{-3}$ $m$ and $70 \times 10^{-3} m$ respectively. The central cylinder is supported on a flexible blade at one end. It is allowed to move in flow normal direction only. The flexible supporting blade is connected to a strain gauge in order to measure the displacement of cylinder.

The tube diameter is $D=12.15 \times 10^{-3} \mathrm{~m}$. The pitch ratio $(p=P / D)$ of the tube arrangement is $p=1.44$ in both (in-flow and flow normal) directions. The modal mass of the cylinder per unit length is $m=0.298 \mathrm{~kg} / \mathrm{m}$. The natural frequency $\left(f_{n}\right)$ and damping ratio $(\zeta)$ of the cylinder in air are $14.39 \mathrm{~Hz}$ and $0.25 \%$ respectively.

The computational domain for LES is $70 \times 10^{-3} \mathrm{~m}$ wide and $48.6 \times 10^{-3} \mathrm{~m}$ deep. The cylinders length is thus $4 D$ against about $8 D$ in the experimental facility. The domain is $269.5 \times 10^{-3} \mathrm{~m}$ long in in-flow direction. The inflow boundary is $5 D$ upstream the tube bundle, while as the outflow is $10 D$ downstream of the tube bundle. The tube diameter $(D)$, array pitch ratio $(p)$ and arrangement of the tube bundle $\left(90^{\circ}\right)$ is identical to the experiment. The geometry of the LES computational domain is shown in Figure 1(a).

The computational domain is descretized in nearly 25.3 million finite volume cells. The mesh near the cylinder surface region is fine enough to resolve the boundary layers of the fluid flow. The first layer of the mesh is placed at a distance of $1.8 \times 10^{-5} \mathrm{~m}$ away from the cylinder surface ensuring the $y^{+}$below 1 . The circumference of each cylinder is discretized in 360 elements, while the 50 cells are placed in spanwise direction. The mesh is coarser $\left(2 \times 10^{-3} \mathrm{~m}\right)$ far upstream and downstream the tube array. Figure 1 (b) shows the details of mesh inside tube array.

In the Large Eddy Simulation (LES) approach of turbulence modeling, the large eddies (bigger than the size of mesh cells) are resolved directly. It contains most of the turbulent energy, however the sub-grid scale turbulence needs to be modeled in order to balance the truncated turbulence energy spectrum. The unfiltered eddies are assumed to be isotropic and they can be modeled by simple Boussinesq type eddy viscosity relations. There exist several models for the sub-grid scale turbulence. The standard Smagarinsky model is considered in this work with an appropriate value of the proportionality constant. However, the choice of sub-grid scale model has little influence on the results (Rollet-Miet et al [15], Benhamadouche and Laurence [17]) of interest.

The filtered Navier-Stokes equations in incompressible form can be written as,

$$
\begin{gathered}
\frac{\partial \tilde{u}_{i}}{\partial x_{i}}=0 \\
\frac{\partial \tilde{u}_{i}}{\partial t}+\tilde{u}_{j} \frac{\partial \tilde{u}_{i}}{\partial x_{j}}=-\frac{1}{\rho} \frac{\partial \tilde{p}}{\partial x_{i}}+\frac{\partial}{\partial x_{j}}\left[\left(v+v_{t}\right)\left(\frac{\partial \tilde{u}_{i}}{\partial x_{j}}+\frac{\partial \tilde{u}_{j}}{\partial x_{i}}\right)\right]
\end{gathered}
$$




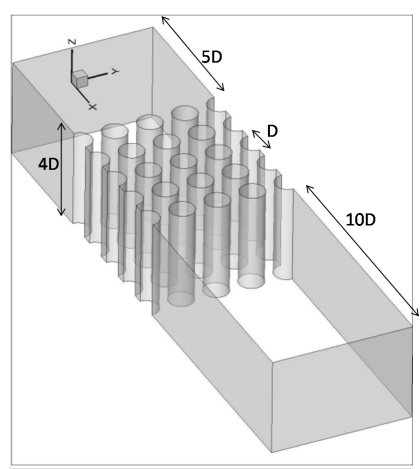

(a) Geometry

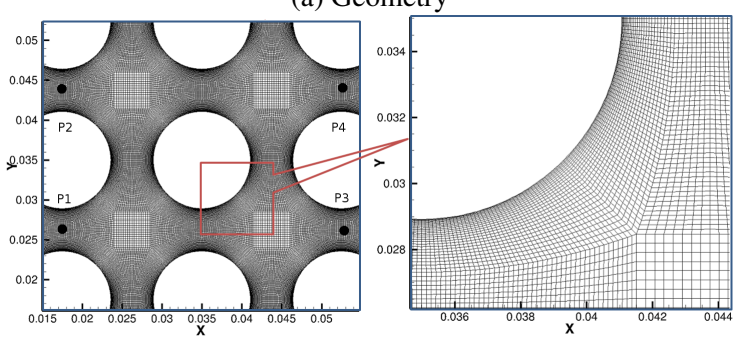

(b) Mesh

Fig. 1: Computational Domain with probes $(P 1, P 2, P 3$ and $P 4)$

Where $\tilde{u}_{i}$ is the filtered instantaneous velocity in $i$ direction. The space and time are represented by $x_{i}$ and $t$ respectively. $\rho$ is the fluid density, while $v, v_{t}$ are the fluid kinematic viscosity and turbulent viscosity respectively. $\tilde{p}$ is the filtered pressure. The subg-grid scale stress tensor is given by Equation (3).

$$
\tau_{i j}=-2 v_{t} \tilde{S}_{i j}+\frac{1}{3} \tau_{k k} \delta_{i j}
$$

The trace term of the sub-grid scale stress tensor is grouped with the pressure $(\tilde{p})$. The value for the turbulent viscosity $v_{t}$ is provided by Smagorinsky's model (Equation 4).

$$
v_{t}=\left(C_{s} l_{g}\right)^{2} \sqrt{2 \tilde{S}_{i j} \tilde{S}_{i j}}
$$

Where, $l_{g}, C_{s}$ are the grid size and Smagorinsky's constant respectively. $\tilde{S}_{i j}$ represents the strain rate tensor. The value of constant $C_{s}$ is about 0.18 for isotropic turbulence at high Reynolds number. It decrease near wall or in shear flows to about 0.1 . The value taken here is 0.065 .

The computations are performed by using Code_saturne, an open-source incompressible Navier-Stokes solver developed by Électricité de France (EDF). It is based on a co-located finite volume method. The second order central difference and Crank-Nicolson schemes are used to perform the space and time discretization 
respectively. A time step has a predictor and a correction steps. In the predictor step all physical properties are calculated along with the velocity field, while as in the correction step the pressure equation is accounted implicitly. The time-step used for all simulations is $2 \times 10^{-4} s$. The simulations are carried out for about $40 s$ each, in order to have reasonable statistics of results.

The cylinder movement is coupled with the fluid flow by the method of ArbitraryLagrangian-Eulerian (ALE). The moving mesh (boundary) is considered in flow equations in terms of the mesh velocity. In response, the forces experted by the fluid flow are used to displace the cylinder boundary surface. In the experiment, the cylinder is rigid and flexibly mounted. An equivalent numerical arrangement is a mass on spring physics, where, $m$ is the mass of cylinder, $k$ and $c$ are the stiffness and damping coefficients of the cylinder oscillations. The equation of motion for the cylinder can be given by,

$$
m \frac{d^{2} y}{d t^{2}}+c \frac{d y}{d t}+k y=F_{y}
$$

Where, $y$ is the displacement of the cylinder in flow normal direction, while as the fluid force in the same direction is represented by $F_{y}$ on the right hand side of equation. The experimental values of modal mass and damping ratio can be used to estimate the stiffness and damping coefficients in air, by using following relations

$$
k=\left(2 \pi f_{n}\right)^{2} m \quad \text { and } \quad c=2 \zeta \sqrt{\mathrm{km}}
$$

The ordinary differential equation (Equation (5)) is numerically solved using Newmark HHT algorithm, in which the fluid forces are used to estimate the displacement $y$. The new position of the cylinder is achieved by solving Poisson's equation for re-meshing before the next flow iteration. The deformation of nearwall mesh is controlled by assigning a high value for an artificial mesh viscosity.

\section{Results and analysis}

\subsection{Results comparison with experiments}

The experiments are performed for Reynolds number $R e_{g}$ ranging from 2000 up to 6000. The Reynolds number is defined using intertube (gap) velocity $u_{g} \mathrm{~m} / \mathrm{s}$ and cylinder diameter $D m$ as $R e_{g}=\left(\rho u_{g} D\right) /(\mu)$, where $\rho$ and $\mu$ are fluid density and dynamic viscosity respectively. The non-dimensional reduced velocity is defined as $u^{*}=\left(u_{g}\right) /\left(f_{n} D\right)$, where $f_{n}$ is the cylinder response frequency in water at a particular flow velocity $\left(u_{g}\right)$. Figure 2 shows the response frequency and damping ratio against the increasing intertube flow velocity. The experimental plots (shown in red color) and the LES simulation plots (shown in green color) show a decreasing trend at the beginning for reduced velocity upto $u^{*} \approx 1.8$. The curves show increasing treads 
from $u^{*} \approx 1.8$ upto $u^{*} \approx 2.10$. It is followed by the decreasing values at the onset of instability for $u^{*} \approx 3.19$.

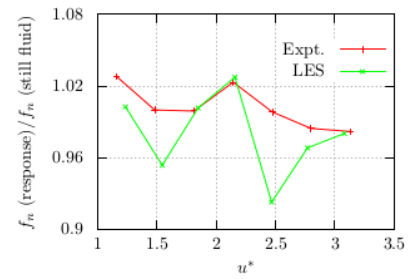

(a) $f_{n}$ versus $u^{*}$

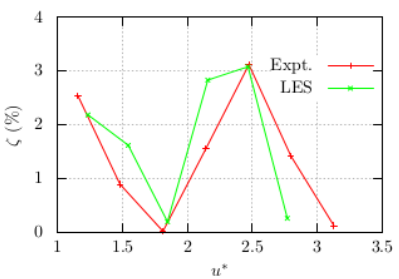

(b) $\zeta$ versus $u^{*}$

Fig. 2: Comparison of the cylinder response frequency $f_{n}$ and damping ratio $\zeta$

The Time Domain Modal Analysis (TDMA) of Poly Reference (PR) type is used to identify the modes and corresponding damping ratios for experimental results, while as the Half Power Bandwith method is suited for LES time response signals. The damping of the cylinder vibrations initially decreases with increase in the gap velocity up to $u_{g} \approx 0.25 \mathrm{~m} / \mathrm{s}\left(u^{*} \approx 1.8\right)$ (see Figure $2(\mathrm{~b})$ ). It is followed by increased values of the damping ratio for reduced velocities $u^{*} \approx 1.8$ up to $u^{*} \approx 2.5$. The damping ratio, then follows a monotonous decrease until reaches zero, onset of the instability, in both the experiments and LES results. The critical reduced velocity predicted by LES computations is $u_{c}^{*} \approx 3.14$, against an experimental value of $u_{c}^{*} \approx$ 3.19 .

\subsection{Results analysis}

In order to understand the development of fluid-elastic instability, a comparison is done between static case Large Eddy Simulations (LES) results with the dynamic case LES computations. The spectra of $\mathrm{Y}$ velocity $\left(u_{2}\right)$ at probe locations $P 1$ and $P 3$ are compared between static and dynamic cases for increasing reduced velocity $\left(u^{*}\right)$. Further, the velocity spectra at these upstream and downstream locations are compared with the spectrum of cylinder vibration (y) in Figure (3). The frequencies are normalized by the natural frequency of the cylinder in still fluid medium. The natural frequency of the cylinder in the still water is $\approx 11.5 \mathrm{~Hz}$. In static case, the red curves in Figures 3(a) and 3(b), the shear layer frequency at gap velocity $u_{g}=0.175 \mathrm{~m} / \mathrm{s}$ is $6.5 \mathrm{~Hz}\left(f_{s h}^{*} \approx 0.5652\right)$. In addition, there are higher harmonics of this frequency in the spectra computed at the downstream location P3. In the dynamic case, the green curves in Figures 3(a) and 3(b), there appears an extra frequency peak at both the upstream and downstream locations. It corresponds to the response frequency of cylinder. On other hand, the response spectrum of cylinder, the blue curve in Figures 3(a) and 3(b)) shows a frequency peak at $6.5 \mathrm{~Hz}$ $\left(f^{*} \approx 0.5652\right)$. Figures $3(\mathrm{c})$ and $3(\mathrm{~d})$ show a similar comparison for the intertube 


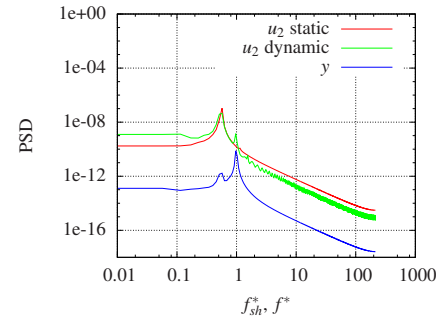

(a) Location $P 1, u_{g}=0.175(\mathrm{~m} / \mathrm{s})$

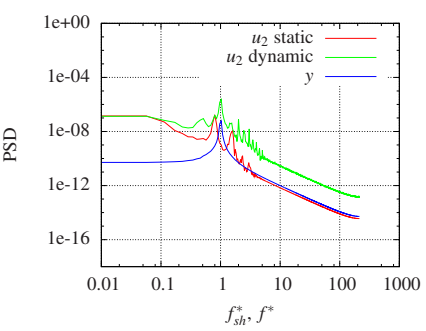

(c) Location $P 1, u_{g}=0.262(\mathrm{~m} / \mathrm{s})$

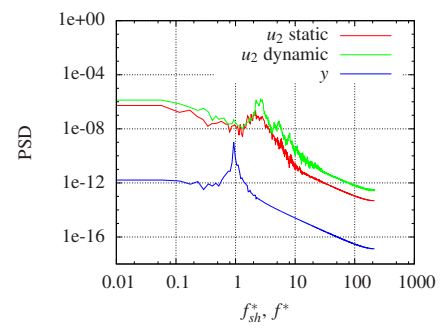

(e) Location $P 1, u_{g}=0.350(\mathrm{~m} / \mathrm{s})$

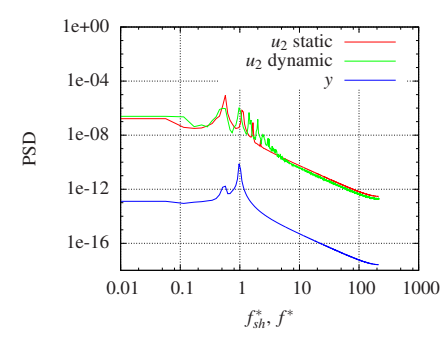

(b) Location $P 3, u_{g}=0.175(\mathrm{~m} / \mathrm{s})$

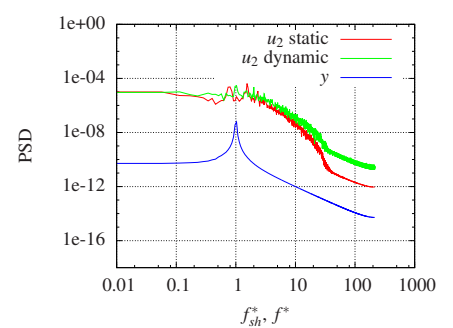

(d) Location $P 3, u_{g}=0.262(\mathrm{~m} / \mathrm{s})$

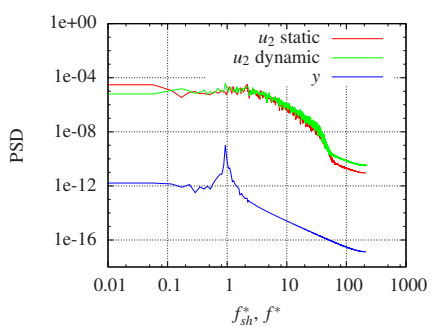

(f) Location $P 3, u_{g}=0.350(\mathrm{~m} / \mathrm{s})$

Fig. 3: Power spectral densities (PSD) of Y velocity in static and dynamic cases at an upstream (P1) and a downstream (P3) locations, in comparison with the cylinder response spectrum

velocity $u_{g}=0.262 \mathrm{~m} / \mathrm{s}$. The red curves of the static case simulation show two frequency peaks, first at $9.2 \mathrm{~Hz}\left(f_{s h}^{*} \approx 0.8\right)$ and second at $\sim 19 \mathrm{~Hz}\left(f_{s h}^{*} \approx 1.652\right)$, at both $P 1$ and $P 2$ locations. On the contrary, the velocity spectra in the dynamic case (green curves in Figures 3(c) and 3(d)) show a distinct frequency at the cylinder response frequency $\left(f^{*} \approx 1\right)$. Further, the power spectral density values are elevated at this velocity $\left(u_{g}=0.262 \mathrm{~m} / \mathrm{s}\right)$, when compared with the other two gap velocities, indicating a possibility of the synchronization between the shear layer frequencies and cylinder response frequency. The flow velocity spectra at gap velocity $u_{g}=0.35$ $\mathrm{m} / \mathrm{s}$ show a wider peak at frequency $f_{s h}^{*} \approx 1.91$, at the upstream $(P 1)$ location only. The frequency peak corresponding to the cylinder vibration do not reflect in the velocity spectra (Figures 3(e) and 3(f)). The shear layer frequencies increase with a further increase in the Reynolds number. The fluid-elastic instability in LES cal- 
culations occurs at Reynolds number $R e_{g}=5310$, where the flow frequencies at upstream locations $(P 1, P 2)$ are about $\sim 3.35$. There is no distinct frequency peak at downstream locations $(P 3, P 4)$. Thus, the time periodicity in flow is less likely the cause of fluid-elastic instability.

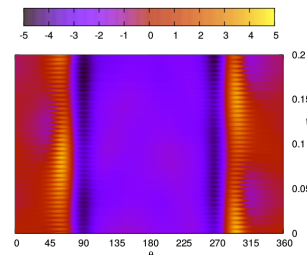

(a) $u_{g}=0.175(\mathrm{~m} / \mathrm{s})$ static

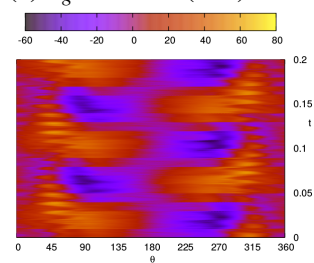

(d) $u_{g}=0.262(\mathrm{~m} / \mathrm{s})$ dynamic

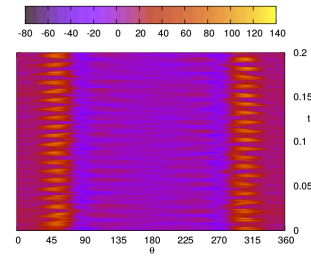

(g) $u_{g}=0.437(\mathrm{~m} / \mathrm{s})$ static

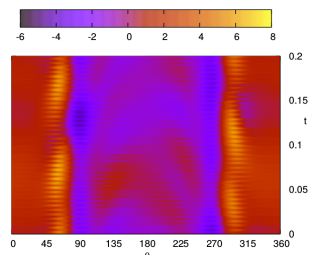

(b) $u_{g}=0.175(\mathrm{~m} / \mathrm{s})$ dynamic

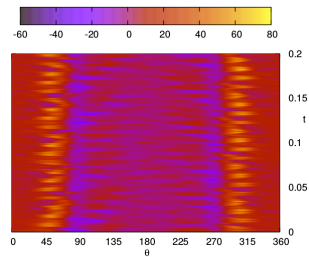

(e) $u_{g}=0.350(\mathrm{~m} / \mathrm{s})$ static

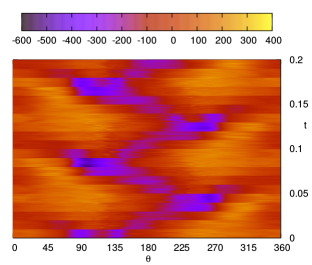

(h) $u_{g}=0.437(\mathrm{~m} / \mathrm{s})$ dynamic

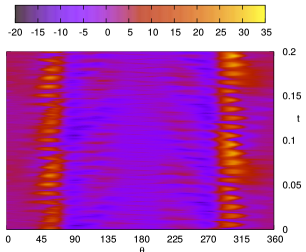

(c) $u_{g}=0.262(\mathrm{~m} / \mathrm{s})$ static

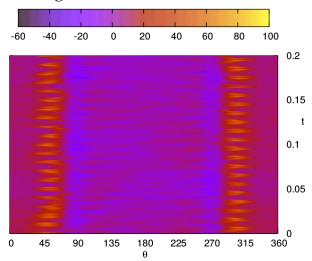

(f) $u_{g}=0.350(\mathrm{~m} / \mathrm{s})$ dynamic

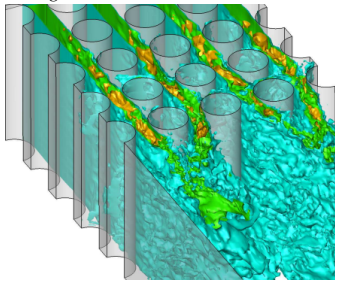

(i) Iso-surfaces velocity plot

Fig. 4: Comparison of time evolving instantaneous surface pressure between static and dynamic cases $(\mathrm{a}-\mathrm{h})$ and flow visualization (i)

The interactions between the cylinder and its adjacent flow streams can be monitored at the interface, the cylinder surface, by means of the fluid forces. The pressure force constitutes major part of the fluid force, even at these low Reynolds numbers $\left(R e_{g} \approx 6000\right)$. The time evolution of pressure profiles on cylinder surface in static and dynamic configurations is presented in Figure (4). The time duration considered on $y$ axis is $0.2 s$, which approximately corresponds to two periods of the cylinder frequency in water $\left(f_{n} \approx 11.5 \mathrm{~Hz}\right)$. In all static case configurations (Figures 4(a), 4(c), 4(e) and 4(g)), the pressure profiles evolve symmetrically in time with respect to the azimuthal angle $\theta=180^{\circ}$. The pressure profile in static case at intertube velocity $u_{g}=0.175 \mathrm{~m} / \mathrm{s}$ (Figure 4(a)), when compared with the dynamic case (figure 4(b)) at same intertube velocity shows more or less symmetrical time evolution with $\sim 40 \%$ increase in the pressure drop in dynamic case. The time-pressure profiles in static and dynamic cases at gap velocity $u_{g}=0.262 \mathrm{~m} / \mathrm{s}$ are compared in Figures 
4(c) and 4(d) respectively. It shows a considerable difference in the time-evolution and the value of pressure drop. The pressure difference in dynamic case has increased by $\sim 3$ times the static case. The pressure time evolution is changed from symmetrical to nearly anti-symmetric with respect to $\theta=180^{\circ}$ location. It indicates that, one flow stream adjacent to the cylinder $\left(\theta=0^{\circ}\right.$ to $\left.\theta=180^{\circ}\right)$ when exerts positive pressure on the cylinder, the other flow stream $\left(\theta=180^{\circ}\right.$ to $\left.\theta=360^{\circ}\right)$ exerts negative pressure on the cylinder surface. Further increase in intertube velocity to $u_{g}=0.35 \mathrm{~m} / \mathrm{s}$, results in a symmetry of the time-evolving pressure profile in dynamic case, similar to the static case for the same Reynolds number (Figures 4(f) and 4(f) respectively). The value of instantaneous pressure drop is $\sim 14 \%$ higher in dynamic case. The cylinder oscillations become unstable at intertube velocity $u_{g}=0.437 \mathrm{~m} / \mathrm{s}$. The transient development of the pressure profile in dynamic case is shown in Figure 4(h). The pressure profile is antisymmetric with sudden increase in pressure drop value (to $1000 \mathrm{~Pa}$ ) against the symmetrical pressure profile in static case (Figure 4(g)) with pressure drop of $220 \mathrm{~Pa}$. The dynamic interaction between the cylinder and adjacent flow streams results in changing the time-pressure profile on cylinder surface for increasing intertube velocity (Figures 4(b), 4(d), 4(f) and 4(h)). A flow visualization is provided in Figure 4(i), in terms of the iso-surface plots for three values of velocity.

\section{Conclusion}

Large Eddy Simulation (LES) carried out to study fluid structure interaction in an inline cylinder array. An Arbitrary-Lagrangian-Eulerian (ALE) approach is adapted to simulate the coupling of fluid flow and motion of cylinder. The response frequency $\left(f_{n}\right)$ and damping ratio $(\zeta)$ of the cylinder for the range of reduced velocities are in agreement with the experimental values. This shows, the dynamic unsteady interactions between fluid load and cylinder vibration are well captured by LES. In the analysis, we shed some light on the dynamic interactions of the cylinder vibration and the adjacent flow streams. The time evolution of the pressure profiles on the cylinder surface is indeed linked with flow stream perturbations induced by the cylinder vibration.

Acknowledgements The authors acknowledge Centre National de la Recherche Scientifique (CNRS) for facilitating the work via Agence Nationale de la Recherche (ANR) project Baresafe.

\section{References}

1. A. Khalifa et al., 2012. A single flexible tube in a rigid array as a model for fluidelastic instability in tube bundles. J. of Fluids and Structures 34, 14-32.

2. A. Khalifa et al., 2013. Modeling of the phase lag causing fluidelastic instability in a parallel triangular tube array. J. of Fluids and Structures 43, 371-384. 
3. B. Anderson et al. 2014. Modeling of fluidelastic instability in a square inline tube array including the boundary layer effect. J. of Fluids and Structures 48, 362-375.

4. B. W. Roberts, 1966. Low frequency aeroelastic vibrations in a cascade of circular cylinders. Mechanical Engineering Science No. 4.

5. Blevins, R., 1974. Fluid-elastic whirling of a tube row. J. of Pressure Vessel Tech. 96, 263-267.

6. C. Liang, G. Papadakis 2007. Large eddy simulation of cross-flow through a staggered tube bundle at subcritical Reynolds number. J. of Fluids and Structures 23, 1215-1230.

7. Connors H.J., 1970. Flow induced vibration of Heat Exchanges, pp 42-56. New York:ASME Fluidelastic vibrations of tube arrays excited by cross flow.

8. Granger, S., and M. P. Paidoussis. An improvement to the quasi-steady model with application to cross-flow-induced vibration of tube arrays. Journal of Fluid Mechanics 320 (1996): 163184.

9. H. Tanaka, S. Takahara, 1981. Fluid elastic vibration of tube array in cross flow. J. of Sound and Vibration 77(1), 19-37.

10. J. H. Lever, D. S. Weaver, 1982. A theoretical model for fluid-elastic instability in heat exchanger tube bundles. J. of Pressure Vessel Tech. 104, 147-158.

11. Jus Y., Longatte E., Chassaing J.-C., et al., 2014. Low Mass-Damping Vortex-Induced Vibrations of a Single Cylinder at Moderate Reynolds Number J. Pressure Vessel Technol. 136(5), 051305 .

12. Longatte, E. et al., 2013. Advanced numerical methods for uncertainty reduction on prediction of heat exchanger dynamic stability limits : review and perpectives. Nuclear Engineering and Design 258, 164-175.

13. Paidoussis, M. P., Price, S. J., 1988. The mechanisms underlying flow-induced instabilities of cylinder arrays in crossflow. J. of Fluid Mechanics 187, 45-59.

14. N. K.-R. Kevlahan, 2011. The role of vortex wake dynalics in the flow-induced vibration of tube arrays. J. of Fluids and Structures 27, 829-837.

15. P. Rollet-Miet et al. 1999. LES and RANS of turbulent flow in tube bundles. Int. J. Heat and Fluid Flow 20, 241-254.

16. Price, S. J., 1995. A review of theoretical models for fluid-elastic instability of cylinder arrays in cross-flow. J. of Fluids and Structures 9, 463-518.

17. S. Benhamadouche, D. Laurence, 2003. LES, coarse LES, and transient RANS comparisons on the flow across a tube bundle. Int. J. Heat and Fluid Flow 24, 470-479.

18. S. J. Price and M. P. Paidoussis, 1989. The flow-induced response of a single flexible cylinder in an in-line array of rigid cylinders. J. of Fluids and Structures 3, 61-82.

19. S.S. Chen, 1983a. Instability mechanisms and stability criteria of a group of circular cylinders subjected to cross flow. I. Theory. J. of Vibration, Acoustics, Stress and Reliability in Design 105, 51-58.

20. S.S. Chen, 1983b. Instability mechanisms and stability criteria of a group of circular cylinders subjected to cross flow. II. Numerical results and discussion. J. of Vibration, Acoustics, Stress and Reliability in Design 105, 253-260

21. S. S. Paul et al., 2008. Experimental and numerical investigation of turbulent cross-flow in a staggered tube bundle. Int. J. Heat and Fluid Flow 29,387-414.

22. V. Shinde et al. 2014. Numerical simulation of the fluid structure interaction in a tube array under cross flow at moderate and high Reynolds number. Journal of Fluids and Structures 47, 99-113.

23. Y. A. Hassan, H. R. Barsamian 2004. Tube bundle flows with the large eddy simulation technique in curvilinear coordinates. International Journal of Heat and Mass Transfer 47, 30573071.

24. H. R. Barsamian, Y. A. Hassan 1997. Large eddy simulation of turbulent crossflow in tube bundles. Nuclear Engineering and Design 172, 103-122.

25. Weaver, D., 2008. Some thoughts on the elusive mechanism of fluid elastic instability in heat exchanger tube arrays. flow-induced vibration. In: The 9th international conference on flowinduced vibration. Prague, Czech Republic. 